\title{
Temporal Heterogeneity of ROS1 Fusion and Braf Fusion Conferred Resistance to First-and Third- generation EGFR Tkis: a Case Report.
}

\section{Meng Zhang}

first affiliated hospital of Guangzhou Medical university

\section{Yihua Huang}

Sun Yat-sen University Cancer Center

\section{Baorong Chen}

first affiliated hospital of Guangzhou Medical University

\section{Yuna Peng}

first affiliated hospital of Guangzhou Medical University

Jing Lin

Burning Rock Biotech

\section{Yuan Li}

Burning Rock Biotech

\section{Shuyin Chen}

Burning Rock Biotech

\section{Liya Guan}

Burning Rock Biotech

Guohua Yao ( $\nabla$ yaoguohua999@163.com )

First Affiliated Hospital of Guangzhou Medical College https://orcid.org/0000-0001-5749-3369

Wenfeng Fang ( $\sim$ fangwf@sysucc.org.cn )

Sun Yat-sen University Cancer Center

\section{Case Report}

Keywords: NSCLC, EGFR-TKI, resistance, ROS1 fusion, BRAF fusion, case report

Posted Date: June 12th, 2020

DOI: https://doi.org/10.21203/rs.3.rs-32711/v1

License: (1) (1) This work is licensed under a Creative Commons Attribution 4.0 International License.

Read Full License 


\section{Abstract}

Background: The development of EGFR-tyrosine kinase inhibitors (EGFR-TKIs) have revolutionized the treatment paradigms for non-small cell lung cancer (NSCLC) patients. However, acquired resistance inevitably develops with a median progression-free survival (PFS) less than a year. The advancements in sequencing technologies have significantly promoted the identification of potential resistant mechanisms to targeted therapies.

Case presentation: A 39-year-old Chinese male was diagnosed stage IVB NSCLC with EGFR exon 19 deletion (EGFR 19del) and received icotinib but got primary EGFR-TKI resistance caused by ROS1 fusion one month later. Then he received icotinib plus crizotinib and achieved partial response with a profession free survival of 4.8 months. Then he switched to osimertinib plus crizotinib due to the emergence of EGFR T790M mutation. TRIM24-BRAF fusion was detected after osimertinib failure, which might be potential resistant mechanism to osimertinib.

Conclusions: This is the first case to confirm crizotinib plus icotinib in patients harboring both EGFR $19 \mathrm{del}$ and ROS1 fusion. This case displays the temporal heterogeneity of different resistant mechanisms emerging during disease course, highlighting the importance of dynamic genetic monitoring for better clinical managements.

\section{Background}

The identification of EGFR mutations and the subsequent development of EGFR TKIs have revolutionized the treatment paradigms for NSCLC patients. However, primary resistance to EGFR TKIs exists in a subset of EGFR-mutant patients and acquired resistance inevitably develops with a median progression-free survival (PFS) less than a year ${ }^{1}$. The rising genetic sequencing has contributed to the detection of potential resistant mechanisms. Herein, we reported that different resistant mechanisms including ROS1 fusion, EGFR T790M, BRAF fusion were detected by multiple genetic examinations during the treatment process of an EGFR mutated NSCLC patient, highlighting the importance of dynamic genetic monitoring to tailor precision therapies.

\section{Case presentation}

A 39-year-old Chinese male never-smoker referred to a local hospital for an enlarged lymph node in the right neck in July, 2018. Biopsy of the lymph node led to the diagnosis of metastatic adenocarcinoma of lung origin (T4N3M1b stage IVB). PET-CT revealed a pulmonary mass in the left upper lobe, multiple metastatic lesions in lungs, lymph nodes and skeletons. Blood-based targeted next generation sequencing (NGS) covering 13 oncogenic drivers identified EGFR E746_A750del, an EGFR exon 19 deletion (EGFR 19del). Thereafter, the patient was administered with icotinib as a single agent 125mg daily as first-line treatment on August 13th, 2018. However, the patient presented with an enlarged nodule in the left arm during the treatment. CT after one month demonstrated obvious shrinkage in the lung 
mass but increased lymph nodes in bilateral axilla (Fig.1). Aspiration biopsy of the arm lesion and subsequent amplification refractory mutation system-polymerase chain reaction for EGFR/ALK/ROS1 mutations confirmed metastatic lung adenocarcinoma with ROS1 fusion and EGFR 19del. Based on the tumor genotyping, the patient received orally crizotinib $250 \mathrm{mg}$ and icotinib $125 \mathrm{mg}$ daily since October 19th, 2018. CT after one month of combination therapy showed remarkable shrinkage in lymph nodes and stability in the pulmonary mass, contributing to partial response (Fig.1). All treatment-related adverse events such as grade I rash were tolerable. Considering that magnetic resonance imaging after four months (February 28, 2019) showed an apparent increase in cerebral lesions (Fig.1), the patient underwent whole brain palliative radiotherapy from March 5 to March 19, 2019. EGFR analysis on blood sample identified the emergence of EGFR T790M with EGFR 19del during radiotherapy. Thus, the patient was transferred to osimertinib $80 \mathrm{mg}$ plus crizotinib 250mg daily on March 13,2019 . However, CT after one month of osimertinib plus crizotinib demonstrated worsening pleural effusion and increased lesions in both lungs. NGS covering 520 genes on blood detected the occurrence of TRIM24-BRAF fusion (Fig. 2), EGFR E746_A750del and TP53 mutation, without EGFR T790M (Burning Rock Biotech). The patient was treated with chemotherapy of paclitaxel combined with nedaplatin for 2 cycles. Then the patient refused further treatment and passed away two days after leaving the hospital on August 15, 2019.

\section{Discussion And Conclusions}

ROS1 fusions occur in approximately 1-2 \% of NSCLC patients and are rarely detected in EGFR mutant patients ${ }^{2}$. Although a recent case has reported that combined crizotinib with osimertinib could overcome GOPC-ROS1 rearrangement caused by osimertinib resistance, there are limited studies about targeted therapies for patients with both EGFR and ROS1 mutations ${ }^{3}$. Herein, we reported that a NSCLC patient with primary EGFR-TKI resistance caused by ROS1 fusion responded to icotinib plus crizotinib. To the best of our knowledge, our case initially confirms the effect of crizotinib plus icotinib in a NSCLC patient with EGFR and ROS1 mutations, further supporting the feasibility of combination therapy. The patient achieved partial response with a profession free survival of 4.8 months and transferred to osimertinib plus crizotinib due to the emergence of EGFR T790M mutation. Unfortunately, the patient still developed disease progression after one month. TRIM24-BRAF fusion was identified through NGS after osimertinib failure, suggesting a potential resistant mechanism to osimertinib. In vitro study has confirmed that BRAF fusions could confer resistance to osimertinib4. TRIM24-BRAF has been confirmed as an oncogenic driver in lung adenocarcinoma and the corresponding ERK phosphorylation can be suppressed by sorafenib in cell lines ${ }^{5}$. The concomitant TRIM24-BRAF and EGFR T790M might indicate a potent combination therapy deserving further exploration. In conclusion, the temporal heterogeneity of ROS1 fusion and BRAF fusion detected by genetic sequencing emphasizes the necessity of dynamic genetic monitoring for appropriate treatment along treatment process. Furthermore, to the best of our knowledge, our report is the first to suggest antitumor effect of crizotinib plus icotinib in a patient bearing both EGFR 19del and ROS1 fusion. Since preclinical studies has proved the oncogenic activity of TRIM24-BRAF in lung adenocarcinoma, we propose that the acquired TRIM24-BRAF fusion might confer resistance to osimertinib, which hasn't been reported yet. However, the potential therapeutic strategy of targeting BRAF 
fusion to overcome the acquired resistance to osimertinib warrants further studies. Moreover, monitoring of molecular mutation profiling using liquid biopsy or re-biopsy samples is highly recommended since it could guide treatment options after disease progression in EGFR-TKI-treated NSCLC patients.

\section{Abbreviations}

PFS: progression-free survival

EGFR-TKIs: epidermal growth factor receptor- tyrosine kinase inhibitors

NSCLC: non-small cell lung cancer

PET-CT: Positron Emission Tomography-Computed Tomography

CT: Computed Tomography

\section{Declarations}

\section{Acknowledgements}

We owe thanks to the patient who participated in this study and his family. We also thank the whole project team for contributing in this case. This work was financially supported by National Key R\&D Program of China (2016YFC0905500, 2016YFC0905503), Chinese National Natural Science Foundation (81772476, 81602005, 81872499, and 81702283), Science and Technology Program of Guangdong (2017B020227001), Science and Technology Program of Guangzhou (201607020031).

\section{Ethics approval and consent to participate}

Not applicable.

\section{Consent for publication}

Written informed consent for the publication of clinical data and images were obtained from the patient.

\section{Availability of data and materials}

The datasets used and analyzed during the current study are available from the corresponding author on reasonable request.

\section{Competing interests}

The authors declare that they have no competing interests.

\section{Funding}


Not applicable.

\section{Author's contributions}

All authors participated in data collection, data analysis, and final approval of the manuscript.

\section{References}

1. Lee DH. Treatments for EGFR-mutant non-small cell lung cancer (NSCLC): The road to a success, paved with failures. Pharmacol Ther 2017;174:1-21.

2. Bergethon $\mathrm{K}$, Shaw AT, Ou SH, et al. ROS1 rearrangements define a unique molecular class of lung cancers. J Clin Oncol 2012;30:863-870.

3. Zeng L, Yang N, Zhang Y. GOPC-ROS1 Rearrangement as an Acquired Resistance Mechanism to Osimertinib and Responding to Crizotinib Combined Treatments in Lung Adenocarcinoma. J Thorac Oncol 2018;13:e114-e116.

4. Vojnic M, Kubota D, Kurzatkowski C, et al. Acquired BRAF Rearrangements Induce Secondary Resistance to EGFR therapy in EGFR-Mutated Lung Cancers. J Thorac Oncol 2019;14:802-815.

5. Nakaoku T, Tsuta $\mathrm{K}$, Ichikawa $\mathrm{H}$, et al. Druggable oncogene fusions in invasive mucinous lung adenocarcinoma. Clin Cancer Res 2014;20:3087-3093.

\section{Figures}

\section{Figure 1}

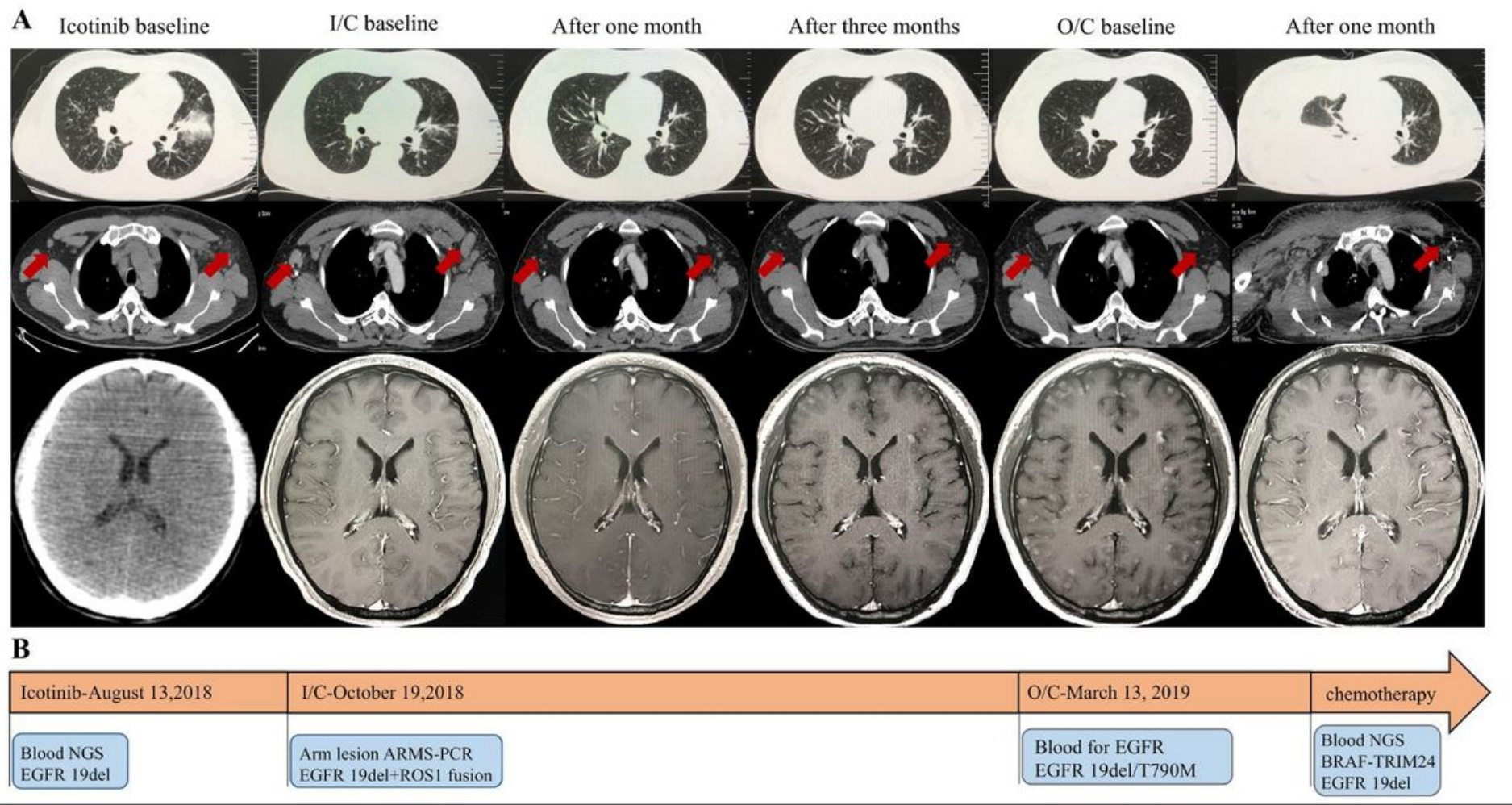


Figure 1

Radiologic images and timeline of the patient's clinical course. A. Clinical response to icotinib, icotinib plus crizotinib, osimertinib plus crizotinib at different time. B. Treatment history and genetic results during the patient's clinical course. I/C indicates icotinib plus crizotinib. O/C indicates osimertinib plus crizotinib.

\section{Figure 2}

A
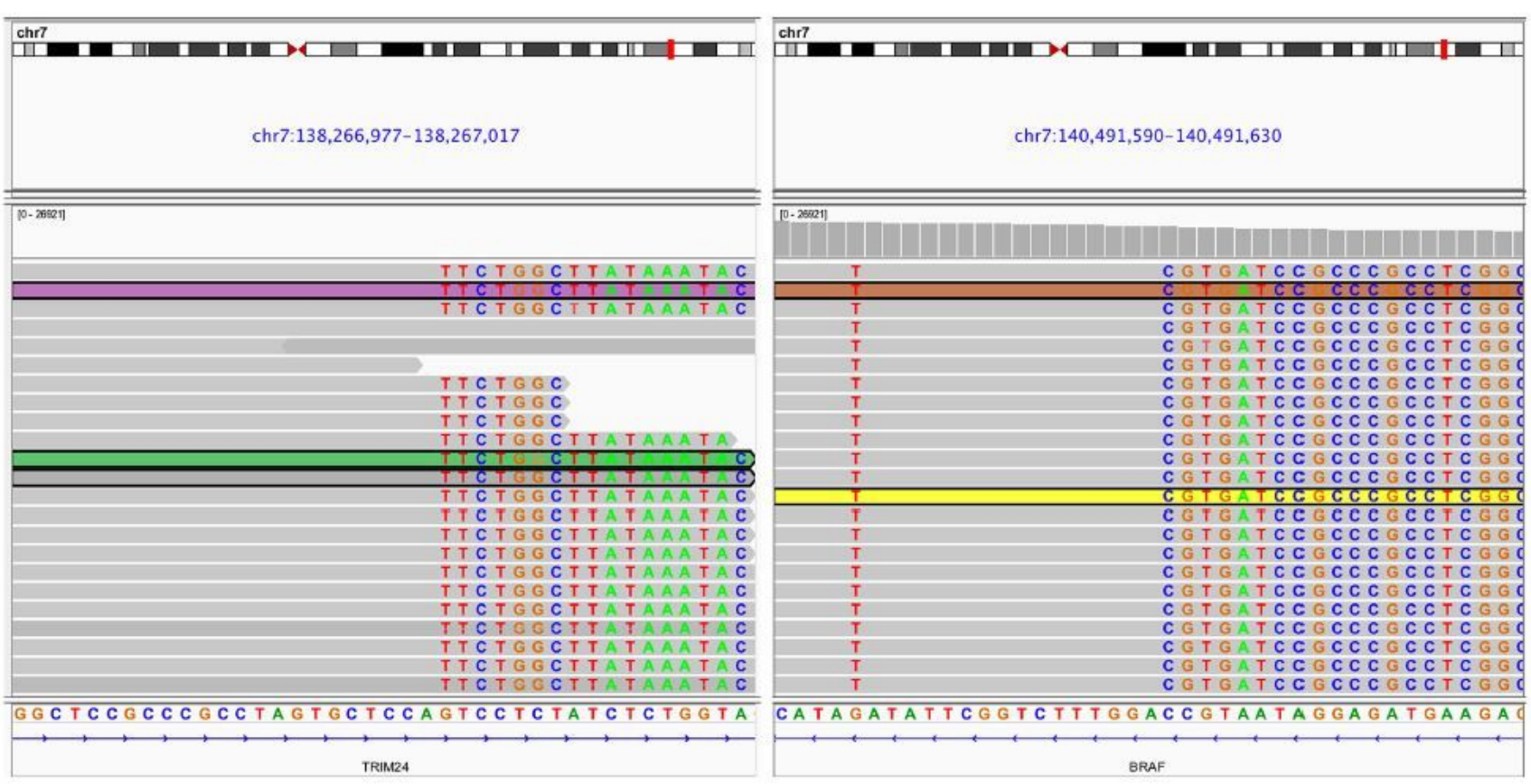

B

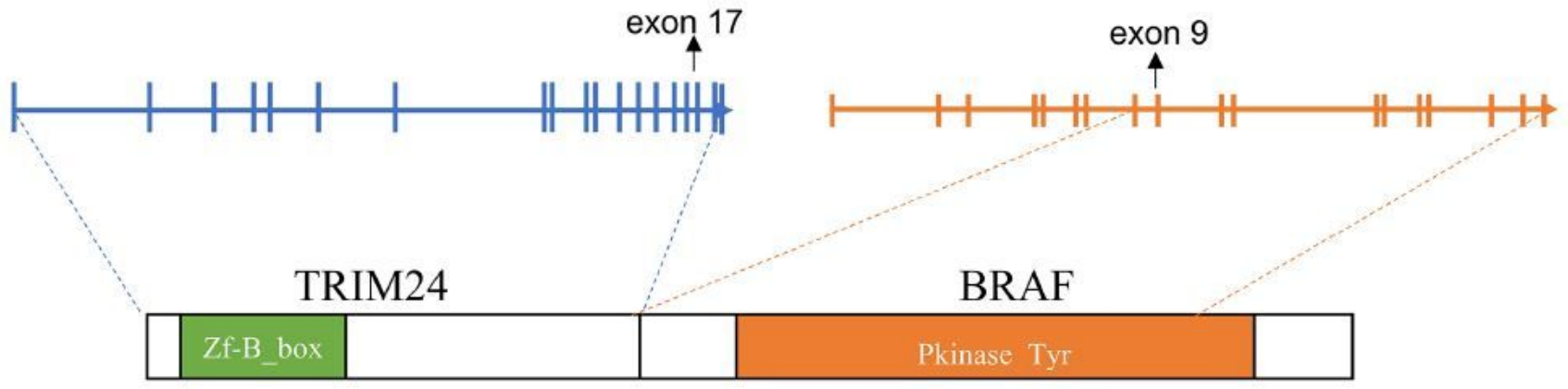

TRIM24-BRAF

\section{Figure 2}

Next-generation sequencing revealed a TRIM24: BRAF fusion (exon17:exon9). A. Screenshot from the Integrative Genomics Viewer demonstrates the chromosomal rearrangement involving TRIM24 (left) and BRAF (right). B. Illustration below demonstrates the protein structure resulting from the gene fusion indicating the breakpoints of the nearby exons.

\section{Supplementary Files}


This is a list of supplementary files associated with this preprint. Click to download.

- supplement6.pdf 